\title{
El aserrío de Dialium guianense (Aubl) Sandwith en Costa Rica
}

\author{
Roy García Sandí ${ }^{1}$ \\ Róger Moya Roque ${ }^{1}$
}

\begin{abstract}
RESUMEN
En el presente trabajo se pretende evaluar la eficiencia de las sierras de cinta o de banda con las características de los dientes más comunes que se utilizan en la industria Costarricense, así también con tratamientos recomendados para maderas duras (dientes calzados con estelita) como en el caso de la especie de Dialium guianense (Aubl) Sandwith ya que no es utilizada en Costa Rica a pesar de su gran abundancia por considerarse madera extremadamente dura y abrasiva. De igual forma se determinó el contenido de sílice en la especie en dos condiciones, trozas en estado húmedo, cerca de su contenido de humedad máximo, y trozas con 6 meses de haber sido cortadas. Los valores de las propiedades físicas y mecánicas también fueron determinados, con el fin de difundir una solución a la problemática en el procesamiento de la especie con los equipos instalados en Costa Rica.
\end{abstract}

PALABRAS CLAVE:

Aserrío, contenido de sílice, Dialium guianense, estelita, moldurado, propiedades físicas y mecánicas.

\begin{abstract}
It is intended here, to evaluate the efficiency of band saws with the characteristics most commonly used in Costa Rica, as well as treatments recommended for hardwoods (teeth with stelite reinforcements) such as is the case of the species Dialium guinense (Aubl) Sandwith since it is not widely used in Costa Rica in spite of its great abundance because it is considered an extremely hard and abrasive wood. At the same time, the sillica content was determined for this species in two conditions: green logs, near its maximum moisture content and logs with almost six months to be cut. The values of the physical and mechanical properties were also determined in order to disseminate a solution to the problems found in the processing of the wood species with the equipment installed in Costa Rica.
\end{abstract}

KEY WORDS:

Sawing, sillica content, Dialium guianense, estellite, moulding, physical and mechanical properties. 


\section{INTRODUCCION}

El Dialium guianense (Aubl) Sandwith conocido en Costa Rica como tamarindo y en México como paque o guapaque, es una especie abundante en los bosques primarios, en los bosques que han sufrido un cierto grado de aprovechamiento forestal, y en los bosques que están en su etapa de regeneración (bosque secundario).

Esta especie presenta dos características importantes, alto valor de peso específico básico $\left(0.83 \mathrm{gr} / \mathrm{cm}^{3}\right)$ y un alto porcentaje de contenido de sílice $(0.73 \%)$ lo que provoca un mayor desafilado de las herramientas de corte en el momento de su procesamiento, tanto en las operaciones de aserrío como en las de fabricación de productos. Esta situación, junto con la gran abundancia de especies comerciales, que generalmente son de fácil trabajabilidad, han provocado que en Costa Rica la especie no haya sido aprovechada comercialmente.

Por otro lado, la industria de aserrío se ha establecido principalmente para procesar maderas de especies comerciales. El tipo de máquinas más utilizadas son sierras de cinta o de banda, con diámetros de los volantes entre 1400 y $1600 \mathrm{~mm}$, con anchos de sierras entre 20 y $25 \mathrm{~cm}$, con calibres de 1.24 y 1.47 $\mathrm{mm}$ (17 y 18 BWG, respectivamente) y pasos de dientes de $38 \mathrm{~mm}$, donde el desempeño de las máquinas en especies como el Dialium guianense (Aubl) Sandwith hace el proceso de industrialización poco eficiente y menos rentable.

Actualmente, en Costa Rica se recurre a especies en el bosque que anteriormente no eran aprovechadas comercialmente por la alta escasez de maderas comerciales debido a la deforestación de sus bosques. En este sentido, en los últimos años algunas especies como el caso del Dialium guianense (Aubl) Sandwith, debido a su abundancia en el bosque, se vuelven especies potencialmente comercializables. Estudios como los de Kleich y Pelz, (1994) han demostrado que los bosques primarios poseen $7.23 \mathrm{~m}^{3} / \mathrm{ha}$, en bosques intervenidos $7.42 \mathrm{~m}^{3} / \mathrm{ha}$ y en bosques secundarios $15.49 \mathrm{~m}^{3} / \mathrm{ha}$ para ser aprovechados, cifras que se consideran altas para los tipos de bosques existentes.

En algunas ocasiones donde las trozas de la especie en estudio han sido aserradas, se utiliza la motosierra con un marco como guía para la obtención de tablas. Este aserrío con motosierra, solamente se efectúa cuando la troza se encuentra totalmente húmeda (casi recién cortado el árbol) y no parcialmente seca o totalmente seca ya que desafila mucho la cadena de la motosierra.

\section{OBJETIVOS}

Ante la situación planteada, se considera necesario llevar a cabo estudios técnicos para determinar las características de la especie y estudios de procesamiento para mejorar los métodos de transformación de ésta y poder orientar los posibles mercados.

\section{METODOLOGIA}

Para el cumplimiento de los objetivos de este trabajo, se tomó una muestra de 8 trozas con un volumen de $21.37 \mathrm{~m}^{3}$ con un diámetro promedio de $62 \mathrm{~cm}$ en un largo de $3.36 \mathrm{~m}$. En el aserrío de las trozas, se aplicó un patrón de corte para obtener de la parte central de la troza las probetas de los ensayos físicos y mecánicos según la norma ASTM D-143 (ASTM, 1993) y de los exteriores de la trozas, piezas con espesores de $12 \mathrm{~mm}$, con el fin hacer una gran cantidad de cortes sobre la troza y de esta manera medir los valores de desgaste de los dientes. 
Los ensayos de aserrío se realizaron en dos diferentes aserraderos. Uno de ellos, el Aserradero de Maderas San Gabriel en San Carlos, provincia de Alajuela (Aserradero 1) y otro, el aserradero del Instituto Tecnológico de Costa Rica (Aserradero 2). Cada uno de ellos representa el tipo de aserraderos que se pueden encontrar en Costa Rica. El primero es utilizado para maderas duras y el segundo para maderas suaves. Las características de cada una de las sierras y los dientes de son mostrados en Tabla 1.

En ambos aserraderos se utilizaron sierras a las cuales se les reforzó la punta de los dientes con estelita, con el fin de aumentar la durabilidad del filo. En el aserradero 2 se utilizó como testigo una sierra sin tratamiento de estelita en la punta del diente. En ambos casos se aseguró que las sierras se encontraran bien tensionadas y alineadas.
En cada una de las sierras se determinó el desgaste sufrido por el diente, utilizando un medidor con subdivisiones de hasta $0.001 \mathrm{~mm}$, colocado sobre un soporte diseñado para ser colocado en el cuerpo de la sierra (Fig. 1). Las mediciones de la altura de los dientes se efectuaron después de cada ocho cortes en una troza. En los cortes de la sierra se midieron los valores de largo y ancho de corte con el fin de determinar el área aserrada e igualmente se tomó el tiempo efectivo de corte que corresponde solamente al tiempo en que la sierra se encuentra cortando la madera. No se contabilizaron los tiempos que tardó el carro en devolverse o los tiempos muertos. Se dejaron de hacer las mediciones en el momento en que se consideraba que la herramienta no estaba en capacidad de efectuar más cortes, notándose en el consumo de amperaje del motor, ya que se colocó un medidor en los cables que conducen la electricidad hacia el motor.

TABLA 1. Características de los aserraderos y las sierras utilizados para determinar la eficiencia en el Dialium guianense (Aubl) Sandwith.

\begin{tabular}{||l|c|c||}
\hline \multicolumn{1}{|c|}{ TIPO DE CARACTERISTICA } & ASERRADERO & ASERRADERO \\
& 1 & 2 \\
\hline Diámetro de la sierra $(\mathrm{mm})$ & 1500 & 1500 \\
\hline Ancho de la sierra $(\mathrm{cm})$ & 25 & 20 \\
\hline Calibre de la sierra $(\mathrm{mm})$ & 1.47 & 1.47 \\
\hline Paso del diente $(\mathrm{mm})$ & 38 & 38 \\
\hline Altura del diente $(\mathrm{mm})$ & 15 & 16 \\
\hline Angulo de corte $\left(^{\circ}\right)$ & 25 & 30 \\
\hline Angulo libre $\left(^{\circ}\right)$ & 17 & 15 \\
\hline Angulo de hierro $\left(^{\circ}\right)$ & 48 & 45 \\
\hline Traba del diente $(\mathrm{mm})$ & 0.75 & 0.43 \\
\hline Velocidad de alimentación $(\mathrm{m} / \mathrm{min})$ & 23 & 4.3 \\
\hline Velocidad de corte $(\mathrm{m} / \mathrm{s})$ & 19 & 30 \\
\hline
\end{tabular}




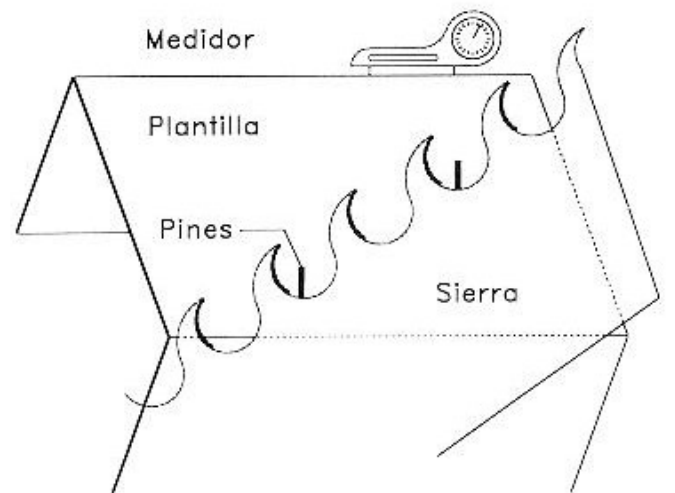

Figura 1. Plantilla para la determinación del desgaste de los dientes

En la elaboración de productos es común encontrar operaciones de cepillado y moldurado por lo que también se determinó el comportamiento de las herramientas en este tipo de operaciones. Para ello, se utilizó una molduradora en la que se usaron tres tipos de cuchillas: de acero de alta velocidad (HSS), reforzados con estelita y reforzados con carburo de tungsteno. En este proceso, simplemente se cuantificó la cantidad de metros lineales cepillados en la molduradora utilizando madera de $7.5 \mathrm{~cm}$ de ancho y el espesor de cepillado que se utilizó fue de $1.5 \mathrm{~mm}$.

Un aspecto que se encuentra muy relacionado con el desafilado de la sierra, es el contenido de sílice presente en la madera. En este estudio se efectuó un análisis de la cantidad presente en la especie Dialium guianense (Aubl) Sandwith. Para este estudio se tomaron 3 trozas de 6.70 metros y a cada una se le cortaron 3 discos de madera de $7.5 \mathrm{~cm}$ de espesor, uno a cada lado de los extremos de las trozas y el último al centro de ella. Los discos de madera se cortaron con un sierra circular de banco para obtener una muestra de aserrín con la que se le determinó el contenido de sílice, tomando como base las normas TAPPI, T257 os76.

Las propiedades mecánicas también fueron determinadas y para ello se empleó la norma ASTM en su designación D-143, parte 1 , volumen 04.09 , tanto para madera seca como para madera verde.

\section{RESULTADOS Y DISCUSION}

Los resultados de desgaste utilizando en el aserrío dientes tratados con estelita fueron menores que cuando se usan sierras sin ningún tipo de tratamiento, ya que presentó menor desgaste por área y una velocidad de desgaste menor (Fig. 2).

El desgaste por área y la velocidad
de desgaste se verán reflejados directamente sobre el área aserrada y el tiempo efectivo de corte de la sierra. Para el caso de la sierra sin tratamiento con estelita el tiempo efectivo de aserrío fue $6.41 \mathrm{~min}$ (sin contar con los tiempos muertos, volteos y otros más en el proceso de aserrío) con una área aserrada de 4.37 $\mathrm{m}^{2}$. Mientras que la sierra con estelita presentó un tiempo efectivo de $21.12 \mathrm{~min}$ en un área de $31.70 \mathrm{~m}^{2}$ (Fig. 3). Tomando como ejemplo una troza de 2.5 metros de longitud y una altura de corte de $25 \mathrm{~cm}$ para el primer caso se harían un total de 7 cortes en un tiempo de aproximadamente 25 min (el tiempo efectivo representa el $25 \%$ del tiempo total de asierre) mientras que con las sierras tratadas con estelita se llegaron a hacer 50 cortes en un tiempo de 1.25 horas, es decir 10 cortes en cada 25 minutos. 


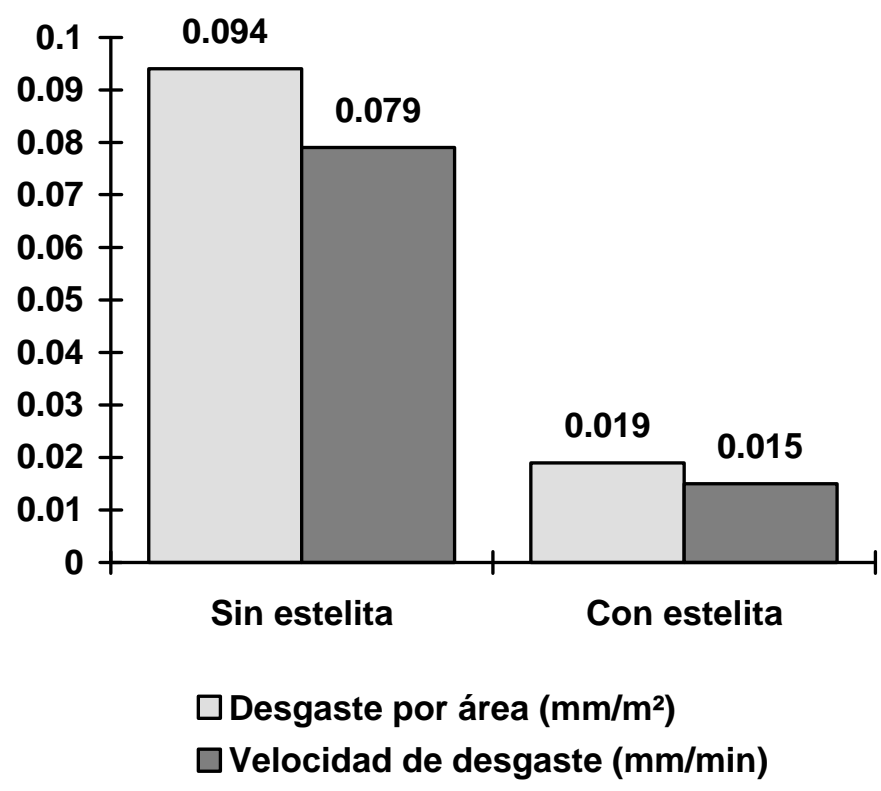

Figura 2. Desgaste por área y velocidad de desgaste para bandas sin tratamiento de estelita y con tratamiento

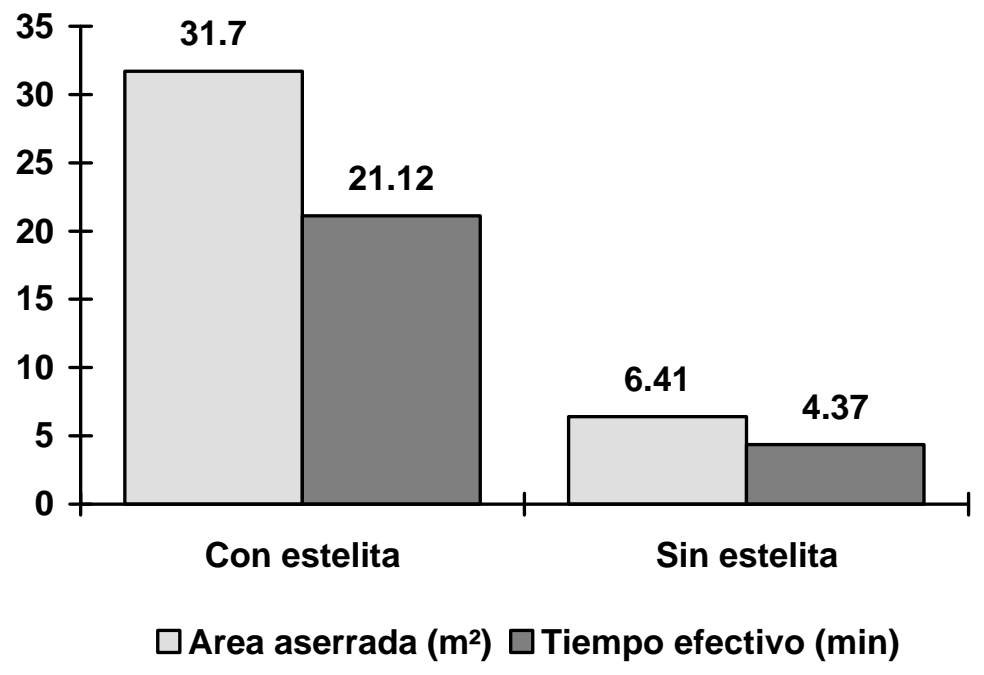

Figura 3. Tiempo efectivo de corte y área aserrada para sierras con tratamiento de estelita y sin estelita 
Con base en los resultados anteriores, se muestra que la especie Dialium guianense (Aubl) Sandwith todavía presenta muchos problemas de procesamiento primario. Usualmente, para el aserrío de una especie con la sierra cinta, ésta debe afilarse cada cuatro horas de trabajo y en este caso con la sierra con estelita solamente se afilaba cada 1.25 horas cuando se utilizó una sierra de banda acondicionada para madera suave. Este tiempo resulta antieconómico para el dueño del aserradero, por lo que debe buscarse otro tipo de alternativas para su procesamiento, como se verá en los siguientes análisis donde se utilizó un aserradero acondicionado para maderas duras.

Los resultados de desgaste por metro cuadrado aserrado y de velocidad de desgaste para el aserradero 1 (maderas duras) y el aserradero 2 (maderas suaves) utilizando sierras calzadas con estelita, son presentados en la figura 4. En la figura se muestra que el desgaste por metro cuadrado es menor en el aserradero 1, pero la velocidad con que se desgasta es mayor que el determinado en el aserradero 2.

En aserradero se usó una velocidad de corte más baja, un ángulo de corte menor y un ancho de la sierra mayor que los utilizados en el aserradero 2 (Tabla 1), lo que permitió trabajar a una mayor velocidad de alimentación. Esta situación permitió, también, hacer una mayor cantidad de cortes, ya que el desgaste por el área aserrada es menor, pero con el inconveniente de sufrir un mayor desgaste del filo con relación al tiempo. Dicho en otras palabras, se corta una mayor cantidad de madera en el aserradero 1 pero tiene que cambiarse la sierra más rápidamente por que ha sufrido un mayor desgaste en el aserradero 2.

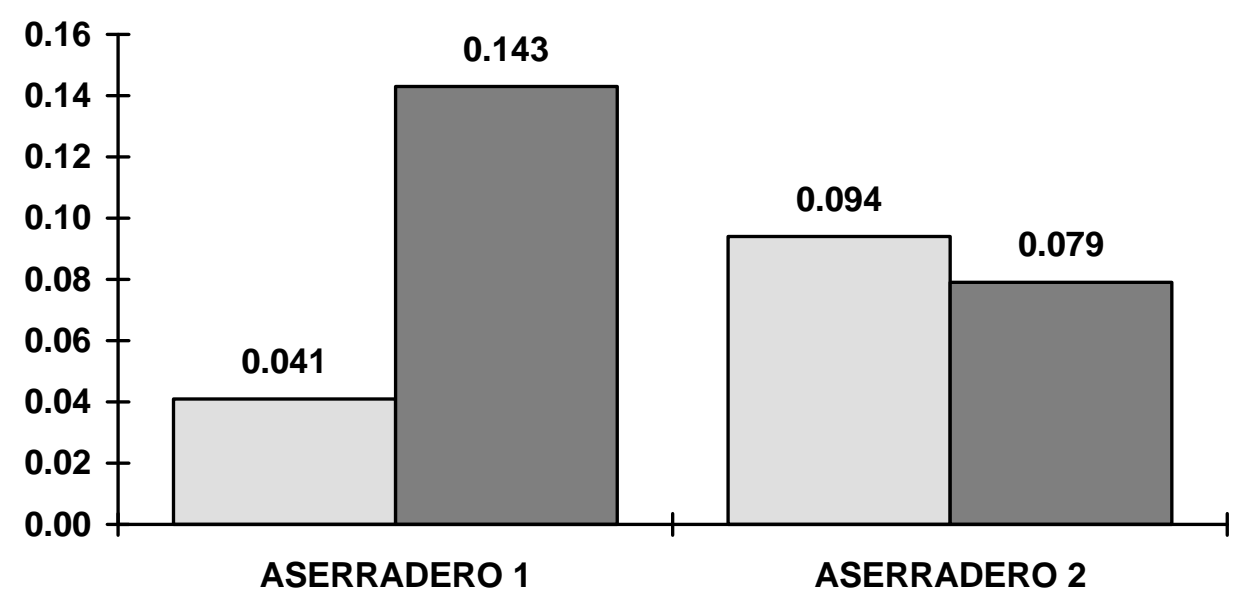

$\square$ Degaste $\left(\mathrm{mm} / \mathrm{m}^{2}\right) \square$ Velocidad de degaste $(\mathrm{mm} / \mathrm{min})$

Figura 4. Desgaste por metro cuadrado aserrado y velocidad de desgaste para los dos tipos de aserraderos en estudio. 
TABLA 2. Condiciones de corte y geometría del diente recomendadas por Ninim con densidad de 0.7 a $0.85 \mathrm{~g} / \mathrm{cm}^{3}$ y utilizadas para madera de Dialium guianense (Aubl) Sandwith

\begin{tabular}{||l|c|c|c||}
\hline \multicolumn{1}{|c|}{ CARACTERISTICA } & RECOMENDADO & ASERRADERO & ASERRADERO \\
& & 1 & 2 \\
\hline Paso de los dientes $(\mathrm{mm})$ & $40-50$ & 38 & 38 \\
\hline Altura de los dientes $(\mathrm{mm})$ & $14-15$ & 15 & 16 \\
\hline Angulo de corte $\left(^{\circ}\right)$ & 25 & 25 & 30 \\
\hline Angulo de hierro $\left(^{\circ}\right)$ & 58 & 48 & 45 \\
\hline Angulo libre $\left(^{\circ}\right)$ & $7-8$ & 17 & 15 \\
\hline Traba $(\mathrm{mm})$ & $0.55-0.60$ & 0.75 & 0.43 \\
\hline Velocidad de la cinta $(\mathrm{m} / \mathrm{s})$ & 25 & 19 & 30 \\
\hline Velocidad de alimentación $(\mathrm{m} / \mathrm{min})$ & $30-40$ & 23 & 4.3 \\
\hline
\end{tabular}

Según Ninim (1989) las condiciones de trabajo y la geometría de los dientes de las sierras para las especie duras con densidades de 0.7 a $0.85 \mathrm{~g} / \mathrm{cm}^{3}$ (el Dialium guianense (Aubl) Sandwith se encuentra dentro de este rango), son las presentadas en la tabla 2, presentándose además las utilizadas en este estudio.

Al comparar las condiciones del aserradero 2 en el aserrío de Dialium guianense, con los valores recomendados para madera duras y abrasivas se observa que no tienen relación o similitud entre ellas, ello dio como resultado un bajo desempeño del aserradero. Mientras que el aserradero 1 tiene condiciones muy parecidas a las recomendadas, lo que provocó, como se analizó en la figura 1, menores valores de desgaste por metro cuadrado aserrado.

Las condiciones del aserradero 2 y las características de los dientes son establecidas para maderas con bajas densidades, lo que provoca un desempeño poco eficiente en el aserrío de la madera de Dialium guianense (Aubl) Sandwith. Los aserraderos existentes en la actualidad en Costa Rica para procesar este tipo de madera tienen que hacer dos cambios básicos, uno de ellos consiste en que se debe modificar la geometría del diente y el otro en que se debe cambiar la velocidad de corte de las sierras para poder cumplir con las recomendaciones dadas por Ninim (1989). Los aserraderos en este medio, usualmente, no procesan un sólo tipo de madera, por lo que para hacer el cambio antes mencionado significaría que el aserradero se dedique exclusivamente para procesar maderas duras.

Las condiciones establecidas para el aserradero 1 son de maderas duras, la diferencia significativa más grande, es el ángulo libre. El valor recomendado es $7-8^{\circ}$ y el utilizado es de $17^{\circ}$, en el caso de modificar este valor lo que se logra, básicamente, es fortalecer el diente aumentando el ángulo de hierro. Al corregir esta situación se vería aumentada la velocidad de alimentación ya que el diente se vuelve más rígido. No se debe pensar que el desafilado del diente sería menor ya que el ángulo de corte siempre se mantiene constante y este ángulo tiene incidencia directa sobre el desafilado de los dientes. 
Con el área aserrada $\left(0.041 \mathrm{~mm} / \mathrm{m}^{2}\right)$ para el aserradero 1 es posible hacer la evaluación de un aserradero que se dedique a procesar el Dialium guianense (Aubl) Sandwith. Para ello se analiza la siguiente condición: se va a aserrar una troza de $50 \mathrm{~cm}$ de diámetro y $2.5 \mathrm{~m}$ de largo, esto significa un área por cada corte de $1.25 \mathrm{~m}^{2}$. Al tener un desgaste de 0.041 $\mathrm{mm}$ por cada metro cuadrado, en la condición anterior el diente tendrá un desgaste de $0.05 \mathrm{~mm}$ en ese $1.25 \mathrm{~m}^{2}$. En 20 cortes en la troza se tendría un desgaste de $1 \mathrm{~mm}$ y en 40 cortes $2 \mathrm{~mm}$; en ese momento la punta del diente tendría una forma casi redonda y se haría necesario nuevamente afilar las sierras.

Basándose en los anteriores análisis es necesario tomar en consideración varios aspectos para poder lograr el aserrío de la especie de Dialium guianense (Aubl) Sandwith con las condiciones existentes en los aserraderos en Costa Rica:

1. En los aserraderos acondicionados para madera duras, se deben utilizar anchos de sierras mayores ya que se tienen mejores resultados que aquellas de bajas dimensiones en el ancho. Las sierras de $25 \mathrm{~cm}$ se desempeñan mejor que las sierras de $20 \mathrm{~cm}$.

2. Se debe modificar la geometría o características de los dientes a las condiciones recomendadas para madera duras según Ninim (1989). Ello no significa que el proceso se hará más rentable, ya que el tiempo efectivo de la sierra no es tan alto para poder mantener la sierra trabajando por varias horas. A pesar de que el aserradero 1, acondicionado para madera dura, dió un menor valor de desgaste por área aserrada que el aserradero 2 (maderas suaves), el desgaste por minuto es menor, lo que significa que el tiempo efectivo de corte es menor que el obtenido en le aserradero 2 que fue de 21 minutos.

3. Basados en el desgaste que sufre una sierra de banda con las condiciones de corte del diente y el tiempo efectivo, se deben hacer pocos cortes en una troza. Esto significa que en una troza se deben obtener, inicialmente, bloques de mayores dimensiones, superiores a los $5 \mathrm{~cm}$ y luego hacer el reaserrío de la especie con sierras circulares con puntas de tungsteno.

Aunque se tengan en consideración los anteriores aspectos, el alto grado de desgaste que se tiene cuando se corta madera de Dialium guianense (Aubl) Sandwith provoca un aumento de los costos en el aserrío ya que constantemente se debe estar cambiando la sierra de banda para afilarse, lo que haría el proceso de aserrío poco rentable con sierras de cinta.

El proceso de cepillado de la madera se llevó a cabo con madera secada parcialmente, ya que el tiempo entre el aserrío y esta operación fue de 2 semanas en que la madera estuvo almacenada sin ningún tipo de apilado.

El comportamiento de la especie en el proceso de cepillado se analizó utilizando tres diferentes tipos de materiales en las cuchillas. Los mejores resultados se obtuvieron con cuchillas de carburo de tungsteno, con las que se procesó una cantidad de 1,500 metros lineales a una velocidad de alimentación de 10 metros por minuto durante un período de 2.5 horas efectivas de trabajo. El otro tipo de material utilizado fueron cuchillas de estelita, con las cuales se procesó una cantidad de 450 metros lineales a una velocidad de 6 metros por minuto en un tiempo de 1.25 horas efectivas de trabajo. El último tipo de material utilizado fue cuchillas de acero de alta velocidad (HSS), donde la cantidad 
procesada fue de 250 metros lineales a una velocidad de 6 metros por minuto en un tiempo efectivo de trabajo de 41 minutos.

En la determinación del porcentaje de sílice presente en la madera de Dialium guianense (Aubl) Sandwith, se encontró que, en una troza con un contenido de humedad de $35 \%$, se obtiene un $0.73 \%$ de sílice presente en la madera; y en una troza con un contenido de humedad de $81 \%$ se presenta un porcentaje de sílice de 0.4 , presente en la madera.

Esto demuestra por qué el aserrío se hace en la montaña con motosierra cuando el árbol esta recién cortado, ya que en la troza verde se presenta menor cantidad de sílice que cuando la madera se encuentra con un menor grado de contenido de humedad. Ya que por un lado aumenta la resistencia de la madera y por otro lado el contenido de sílice.
En el caso del aserrío llevado a cabo en el estudio, las trozas se encontraban húmedas por lo que los resultados obtenidos serían los más bajos, puesto que cuando la madera se encuentre seca los valores de desgaste serán mucho mayores por la mayor presencia de sílice en la madera. En el caso del cepillado, las piezas de madera presentaban cierto grado de sequedad, no llegaban al punto de saturación de las fibra, por lo que cuando se cepille madera totalmente humedad la cantidad de metros procesados serán mayores.

Las propiedades físicas y mecánicas para la especie fueron también determinadas; dentro de los principales resultados se puede observar que el peso específico obtenido es menor al reportado por otros autores (Escobar, 1995). En las tablas 3 y 4 se presentan los valores obtenidos en los ensayos.

TABLA 3. Clasificación del Dialium guianense (Aubl) Sandwith según su peso específico y contracciones

\begin{tabular}{||l|c|c|c|c||}
\hline PARAMETRO & $\begin{array}{c}\text { PESO } \\
\text { ESPECIFICO } \\
\text { BASICO }\end{array}$ & $\begin{array}{c}\text { CONTRACCION } \\
\text { TANGENCIAL } \\
(\%)\end{array}$ & $\begin{array}{c}\text { CONTRACCION } \\
\text { RADIAL } \\
(\%)\end{array}$ & $\begin{array}{c}\text { ESTABILIDAD } \\
\text { DIMENSIONAL }\end{array}$ \\
\hline Valor & 0.83 & 8.3 & 4.96 & 1.67 \\
\hline Clasificación $^{*}$ & muy pesado & baja & baja & estable \\
\hline
\end{tabular}

*Clasificación según Echenique, 1975

TABLA 4. Propiedades mecánicas del Dialium guianense (Aubl) Sandwith en condición verde y seca

\begin{tabular}{||l|c|c||}
\hline \multicolumn{1}{|c|}{ PROPIEDADES MECANICAS } & $\begin{array}{c}\text { CONDICION VERDE } \\
(40 \% \mathrm{CH})\end{array}$ & $\begin{array}{c}\text { CONDICION SECA } \\
(12 \% \mathrm{CH})\end{array}$ \\
\hline Módulo de ruptura en flexión $\left(\mathrm{kg} / \mathrm{cm}^{2}\right)$ & 1073.88 & 1555.99 \\
\hline Módulo de elasticidad en flexión $\left(\mathrm{kg} / \mathrm{cm}^{2}\right)$ & 159339.50 & 210391 \\
\hline Compresión paralela $\left(\mathrm{kg} / \mathrm{cm}^{2}\right)$ & 617.63 & 833.78 \\
\hline Dureza axial $(\mathrm{kg})$ & 1076.80 & 1631.58 \\
\hline Dureza lateral $(\mathrm{kg})$ & 1185 & 1579.67 \\
\hline Cortante tangencial $\left(\mathrm{kg} / \mathrm{cm}^{2}\right)$ & 176.61 & 161.57 \\
\hline Cortante radial $\left(\mathrm{kg} / \mathrm{cm}^{2}\right)$ & 174.14 & 165.24 \\
\hline
\end{tabular}


El peso específico de la especie de Dialium guianense (Aubl) Sandwith, presenta un valor alto, recomendado para maderas de uso estructural y sobre todo de alta resistencia, donde las dimensiones de las piezas sean pesadas con el fin de hacer el menor número de cortes posibles en este tipo de madera.

\section{CONCLUSIONES Y RECOMENDACIONES}

1. En Costa Rica, un grupo de los aserraderos de banda se dedica a procesar maderas suaves y otro grupo a procesar maderas duras. Las condiciones actuales de los dos tipos de sierras utilizadas no se desempeñan adecuadamente para el aserrío del Dialium guianense (Aubl) Sandwith, sobre todo porque no se usa ningún tipo de tratamiento en el diente.

2. Los aserraderos de banda instalados para procesar maderas duras se deben cambiar una serie de condiciones, sobre todo en la preparación de las sierras con el objetivo de poder aserrar el Dialium guianense (Aubl) Sandwith. Estos cambios consisten, básicamente, en utilizar mayores calibres, mayores anchos de sierras y dar la geometría del diente según Ninim (1995) y de esta manera aprovechar su potencial en los bosques de Costa Rica; pero este aserrío conllevará mayores costos del aserrío, ya que se tienen que afilar constantemente las sierras lo que hace, quizás, poco rentable el proceso

3. A pesar de que las sierras y los aserraderos se acondicionen para el aserrío del Dialium guianense (Aubl) Sandwith, se deben utilizar patrones de corte con los que se obtengan piezas de grandes dimensiones, con el fin de que se hagan con la sierra de banda el menor número de cortes posibles.

4. Cuando se requieran piezas con pequeñas dimensiones en la sierra de banda, deben obtenerse bloques y posteriormente hacer el reaserrío con sierras circulares con calzas de carburo de tungsteno en los dientes.

5. La troza debe estar totalmente saturada de agua, esto significa que el tiempo que transcurre entre el momento en que se corte el árbol y su aserrío debe ser de unos pocos días y no de semanas, con el fin de que la cantidad de sílice presente en la madera sea menor y de esta manera necesitar un menor afilado de las sierras

6. Los valores aquí mostrados son los obtenidos en sierras de banda, las sierras circulares por su parte permiten utilizar en los dientes puntas de carburo de tungsteno, lo cual presenta una mayor resistencia al desafilado, por que lo sería de mucha trascendencia realizar las investigaciones respectivas con este tipo de equipo de aserrío y tener de esta manera una mejor alternativa para el procesamiento de la especie de Dialium guianense (Aubl) Sandwith, con el fin de aprovechar la especie que se encuentra con mucha abundancia en muchos de los bosques de Costa Rica.

\section{REFERENCIAS}

American Society of Testing and Material, 1993. Standard Methods of Testing Small Clear Specimens of Timber. ASTM, D-143-83, parte I, volumen 04.09. EUA, p: 198.

Amos, G.1948. Silicius inclusions in wood in relation to marine borer resistence. Journal of Council for 
Scientific and Industrial Research. Australia.190 p.

Echenique M., R. 1975. Caracterización tecnológica de 10 especies, No.1, INIREB-PNIET-CONACYT. México.

Escobar, 0. 1995. Las maderas de Colombia. Fascículo 75 Dialium guianense (Aubl) Sandwith. Centro Colombo Canadiense de la Madera. Colombia.6 p.
Klein, Ch. y D. Pelz.1994. Inventario Forestal de la Región Huetar Norte. Resumen de resultados. Proyecto COSEFORMA. Costa Rica. 26 p.

Ninim, L. 1989. Manual del Grupo Andino para aserrío y afilado de las sierras de cinta y sierras circulares. Junta del Acuerdo de Cartagena. Colombia. 300 p. 